Research Article

\title{
AIoT-Based Smart Bin for Real-Time Monitoring and Management of Solid Waste
}

\author{
Aniqa Bano, ${ }^{1}$ Ikram Ud Din $\mathbb{D}^{1},{ }^{1}$ and Asma A. Al-Huqail $\mathbb{D}^{2}$ \\ ${ }^{1}$ Department of Information Technology, The University of Haripur, Haripur, Pakistan \\ ${ }^{2}$ Department of Botany and Microbiology, College of Science, King Saud University, P.O. Box 2455, Riyadh 11451, Saudi Arabia
}

Correspondence should be addressed to Asma A. Al-Huqail; aalhuqail@ksu.edu.sa

Received 18 November 2020; Revised 8 December 2020; Accepted 17 December 2020; Published 29 December 2020

Academic Editor: Habib Ullah Khan

Copyright (c) 2020 Aniqa Bano et al. This is an open access article distributed under the Creative Commons Attribution License, which permits unrestricted use, distribution, and reproduction in any medium, provided the original work is properly cited.

In the current time, the immense growth in population creates unhygienic environment for the citizen of a society with respect to waste generation. This rapid generation of waste leads to various infectious diseases in the environment. As followed by the traditional municipal system, in our surroundings, we can see over flooding of solid waste in the garbage bins. Solid waste management is a pivotal aspect in traditional systems and it is becoming dangerous in most populated areas. Arduous labor works and costs are required to manage and monitor garbage bins in real time. To maintain the cleanliness of a city and for real-time monitoring of trash bins, a smart bin mechanism (SBM) for smart cities is proposed in this paper, which is based on Artificial Intelligent of Things (AIoT). The SBM works on the 3R concept, that is, Reduce, Recycle, and Reuse. The SBM has the access to get real-time information about each bin and avoid overloading of these bins. The proposed framework reduces the labor cost and saves time and energy of the system. It also reduces the rate of disease infections by keeping the cities clean. Fuzzy logic is used for decision-making in selecting appropriate locations in the cities to install trash bins. The framework is implemented in the multiagent modeling environment, that is, NetLogo.

\section{Introduction}

The significant growth of the Internet is leading to the emergence of new technology, that is, Internet of Things (IoT) [1]. The term IoT was first used by Ashton in 1999 [2]. IoT is the hub of physical devices that are interlinked through the Internet. These physical devices, that is, sensors, RFID tags, and various intelligent nodes, can communicate at any time from anywhere. IoT is the backbone of future communication systems where everything will communicate and share information smartly without human instructions [3]. The interconnected devices are transformed as smart objects, which have computational skills that are used to monitor an environment leading to smart cities.

IoT promotes various application areas, such as smart health, [4-13] smart city, [14, 15], environment monitoring $[16,17]$ smart home, $[18,19]$, traffic management [20-24], smart education system, [25], smart farming, [26, 27], and many others [28-35]. In smart cities, various problems occur when devices communicate with each other; one of the important problems is waste management. The main causes for this problem are the rapid growth of the urban population, high demands for food, and various other factors that are influencing the environment in smart cities. With an increase in population, the management of waste or garbage is a very hectic job to do in the current time. Being a member of the society, every local house, industry, and factory generate some amount of waste on a daily basis. This waste is ultimately collected in waste bins and eventually collected by the municipal vehicles and moved to dumping areas for disposing or recycling processes.

To keep the environment green and clean, monitoring and disposing of waste is very important these days. Improper disposal and poor monitoring of collected waste and waste bins can cause serious damage to human lives. This waste can spread various life-threatening diseases that in turn harm the lives of a whole city and country as well. 
Nowadays, cities are facing various problems, such as small parking spaces [36], waste management, communication barriers in traditional systems, and health issues to name a few $[37,38]$. All these problems directly affect the living of humans in their daily routine lives [39]. To overcome and solve the existing problems, a new concept has emerged in the light of IoT, named smart city [14]. IoT provides various new services in a smart city and develops an intelligent society [40, 41]. In IoT-based smart cities, physical devices interact and provide ease to humans according to their own intelligence [42]. IoT is further divided into various fields presented in Figure 1 [43], which explains the web-based rankings of each area in percentage. Based on the literature study, smart homes and smart cities get the highest rankings, which shows the trends in the modern era of technologies.

The rapid growth in population and generation of daily routine garbage or waste make the environment unhygienic for the citizens. The waste is divided into two types, that is, wet and solid waste [44]. In this paper, the focus is on solid waste management. Therefore, a waste management mechanism is proposed for smart cities, named SBM (smart bin mechanism), in order to sanitize and clean the environment intelligently. It is designed for solid waste management and recycling of waste because waste is recyclable and can be reused. The waste management procedure comprises five steps that include collecting waste, transporting, analyzing and processing, recycling, and disposing [44]. In SBM, smart bins (SB) are installed in the urban areas at different points that store garbage. Primarily, the SBM is designed for real-time monitoring of the garbage collecting points. The proposed system will reduce the labor work, time, and cost that are very high in the traditional garbage collection system.

In SBM, different entities have been used which show their own roles in providing services to the citizens. It has trash bins, trash collecting vehicles, and a central database to keep records of the levels. All the entities and their roles are discussed in Section 3.

1.1. SBM Contributions. Waste management is an emerging era of most populated as well as less populated cities. In IoT, it also has emerged as a field in smart cities. Various prior research has been conducted for collecting waste, but most of the research is server-based or authority monitoring of garbage bins that are installed in public places. These systems act upon receiving requests from garbage bins and sending collecting vehicles toward the requesting bins. This mechanism consumes enough an amount of energy and time on fulfilling of a single request. Therefore, an intelligent edgenode based mechanism is necessary for collecting waste from requesting bins, which consumes less energy and time. The proposed framework is based on edge-nodes, that is, the trash bin. When a trash bin reaches the threshold level, it makes a request directly to the collecting vehicle instead of forwarding the request to any central authority. The proposed framework has a significant role in waste collecting procedures as it consumes low power because of its novel edge-based mechanism. Time consumption is also low in SBM because of the less iteration from the request-to-response procedure.

Some significant contributions of the proposed framework are as follows:

(i) Proposing a smart bin mechanism that is based on IoT technology and applications

(ii) Real-time monitoring of the trash bins in a smart city

(iii) Using trash bins in an effective way to facilitate the municipal department and citizens as well

(iv) Reducing labor cost and optimizing resources

(v) Improving environment goals and cleaning cities with limited resources

The proposed study is novel in terms of real-time monitoring and decision-making using fuzzy logic processing. Fuzzy logic provides the best suitable and less dense site of the city to install trash bins. Two fuzzy parameters are used in fuzzy inference systems, that is, distance from the collecting/dumping zone and access to the trash bins.

The proposed study is beneficial in the future era of modern technologies, where everything will be connected via the Internet and communicate without human interruption. The study supports the smart city concept by providing real-time monitoring for climate change. It provides a decision-making mechanism by using a fuzzy inference system.

The rest of the paper is divided into five sections; that is, literature study is discussed in Section 2, SBM methodology is presented in Section 3, results and discussion are provided in Section 4, the paper is summarized in Section 5, and challenges and future work are elaborated in Section 6.

\section{Literature Study}

For the last few years, many researchers are focusing on IoTbased applications, especially smart city [45]. According to [46], a smart city is an infrastructure where everything is interconnected and can interact with each other. In a smart city, everything is supposed to be smart and intelligent in decision-making ability [47]. A smart city leads to a smart environment [30], smart health [48], smart parking, smart economy, smart administration [49], and smart living of the people [50]. The smart city provides all the better facilities to citizens and assures that there is a clean and green environment for them [51]. To make the environment clean, there should be an effective system for collecting waste. In this section, various research about garbage or waste collection and a better management mechanism for the collected waste is reviewed.

2.1. SWMS. Waste management systems play a vital role in reducing the unhygienic objects from a particular area. To avoid these conditions, a smart waste management system (SWMS) [44] is proposed that is based on IoT technology. The SWMS consists of public garbage collectors with 


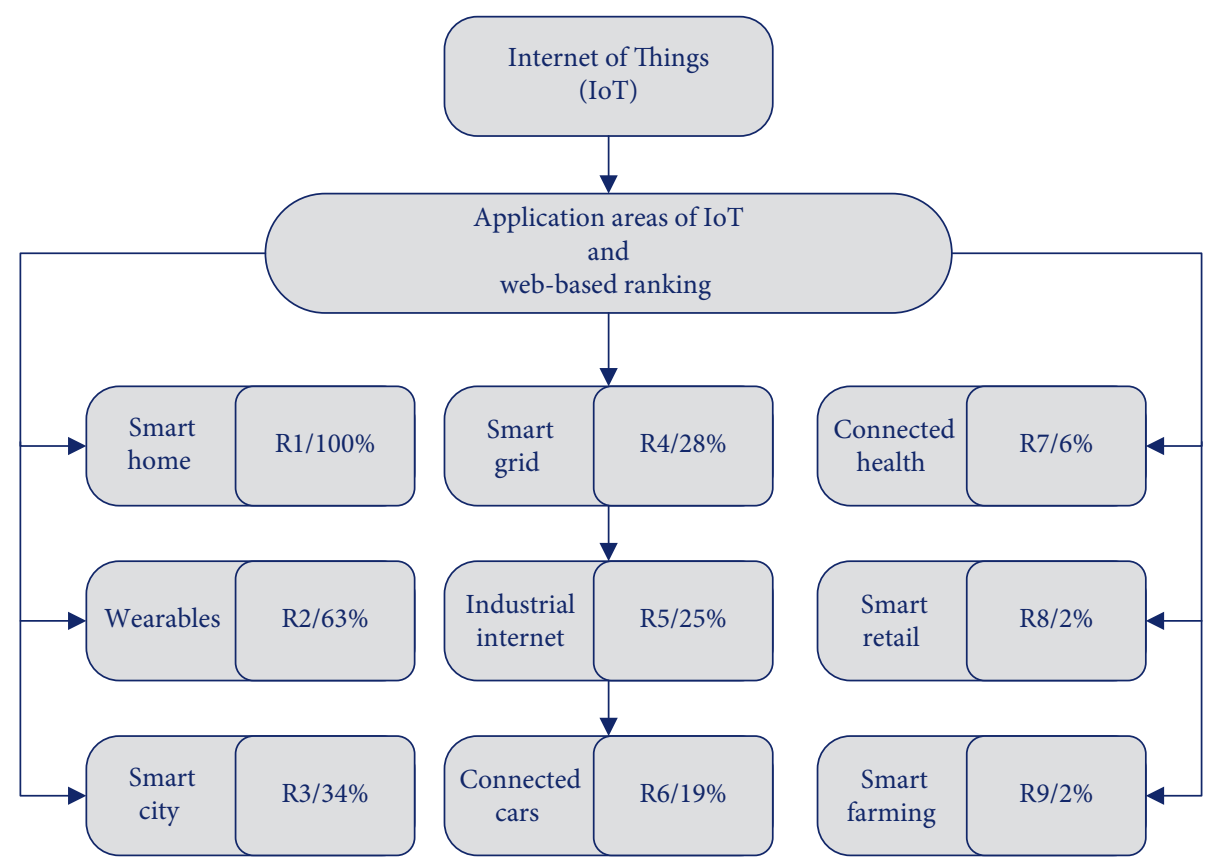

FIgURE 1: Application areas of Internet of Things.

embedded technology that is used to monitor real-time level of garbage bins in public places. Based on the level of garbage bins, an optimized path is selected for the garbage collecting van that eventually reduces fuel cost. The SWMS divides garbage bins into master and slave bins. Each garbage bin is composed of three sensors, such as level sensor, humidity sensor, and load sensor. The master bin is continuously transmitting its data to the cloud with the help of Wi-Fi. Through real-time monitoring, accurate reports can be generated, and, therefore, the efficiency of the system is enhanced.

2.2. GMS. Overflowing of dust bins at public places increases unhygienic environment for the people, especially, in developing countries; this creates serious health problems for the citizens. To cope with these types of situations, an IoT-based garbage monitoring system (GMS) is proposed in [52]. The system contains various dust bins that are distributed in the city. Dust bins transmit the data to concerned authorities in order to clean the garbage. The block diagram of the proposed model consists of two sections, that is, transmitter and receiver sections. The transmitter is installed in dustbins, which is used to transmit collected data from sensors to the receiver end. At the receiver end, the central system receives the data sent from the dustbin and processes it accordingly. The authors used Raspberry $\mathrm{Pi}, \mathrm{RF}$ receiver, and a web browser to fulfil the requirements of the system. The proposed system has some limitations in terms of lacking in reliability of communications among different modules.

2.3. IoT-Based SWM. Waste management is an important service provided by smart cities and supported by IoT. An enhanced system for waste management is proposed in [53] by considering the growth of the population in urban areas. The proposed model mainly consists of four entities, such as smart bins, waste areas, management centers, and collecting trucks, as shown in Figure 2. Statistical analysis and decision-making are successfully done based on the data provided by the above-mentioned entities. The authors stated that the proposed model overcomes the existing issues in the waste collection process, that is, location issues, cleaning costs, health hazards, and many others related to waste management.

2.4. SWM by K-Query Scheduling. An IoT-based system is proposed in [54] that is used for waste management with the help of K-Query scheduling. The system is composed of microcontroller module, GPS module, and ultrasonic sensor. These modules are installed in trash cans. The sensors are used to monitor the trash cans. When a trash can reach an appropriate level, the sensor calculates the level and transmits it to the cloud through Internet. The K-Query scheduling is used to store threshold values in a table created in the MySQL database. The architecture of the system is shown in Figure 3. The K-Query is helpful to reduce unknown entries in the database. For a shorter path, a code with a map and location point is executed only one time. There is no need to execute the code in order to find the route for every event. This system is helpful in reducing manpower used in collecting waste from different locations using manual systems. However, the system has lacking with respect to power interruption.

2.5. SWC as a Service. An IoT-enabled solid waste management system is proposed in [55] for monitoring garbage bin and dynamic routing of the garbage collectors. The proposed system consists of an embedded device for real- 


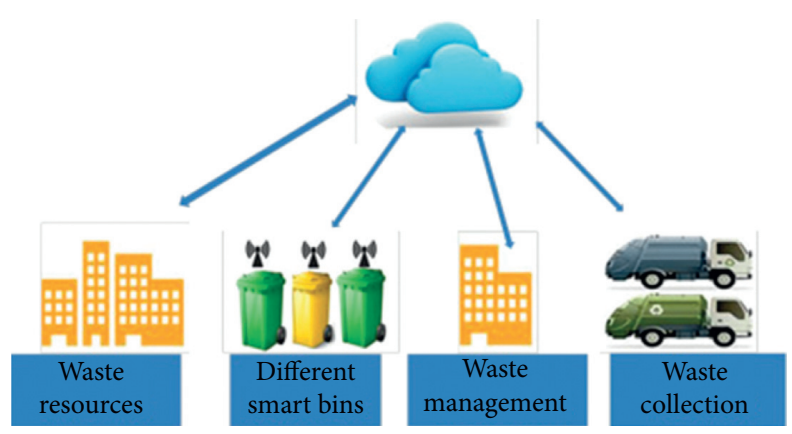

FIgURE 2: Architecture of IoT-based SWM.

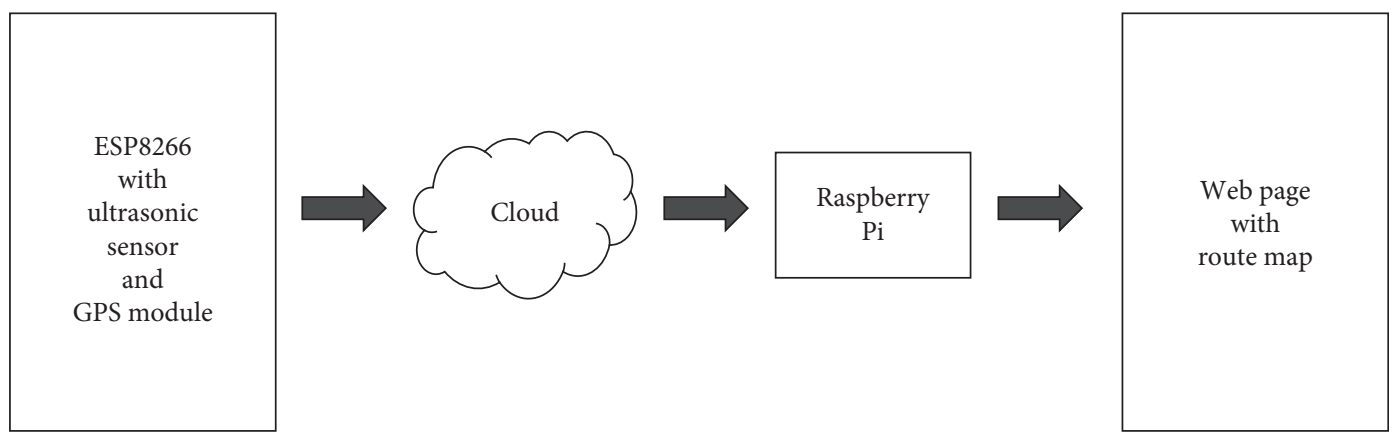

FIGURE 3: Architecture of the K-Query SWM.

time monitoring and scheduling of routes for garbage trucks. The architecture of the proposed system is presented in Figure 4. A mobile application is also designed for the truck driver to handle the data coming from garbage bins and further transmitting it to the cloud. In the proposed system, two garbage bins are installed in one place and solid waste is segregated from dry and wet garbage bins. The system is efficient in decision-making as it uses the GPS module and Google Map API for finding the optimal route to reach the garbage bins.

2.6. SCGCMS. In SCGCMS [56], a smart waste management and monitoring system is proposed for public waste collection that is based on IoT technology. The system consists of two phases where, in phase one, dustbins are installed in different locations and filled randomly while, in phase two, the route for collecting vans is decided on the basis of the dustbin filling ratio. The filling threshold is defined by the authors, which is $10 \mathrm{~cm}$ per dustbin. The system architecture of SCGCMS is shown in Figure 5. In this system, genetic algorithms are used for gathering waste. The dustbins are composed of a weight sensor and Raspberry pi Uno board that is connected with GSM modem and ultrasonic sensor for communications.

2.7. ML-Based WMS. With the rapid growth of IoT and its applications, various critical issues have appeared in todays' lifestyle. One of the most critical issues is waste management in urban areas. To reduce these types of issues, a waste management system [57] is designed for a campus of the Ton
Duc Thang University in Vietnam, which is based on machine learning (ML) in the IoT environment. The authors used graph theory and ML that provide optimal path selection for waste collection on predicting the probability of garbage in trash bins. The proposed system is used for realtime monitoring through integrating multichoices, that is, ultrasound distance, E32 TTL-100 $433 \mathrm{MHz}$ with the LoRa spread-spectrum technology. Energy supply to each node in the network is provided by different sources, that is, solar and batteries. The proposed system for waste collection is better than the existing systems in terms of optimal pathfinding and flexibility.

Table 1 presents the advantages and limitations of the surveyed schemes.

\section{Proposed Methodology}

In light of IoT technology, waste management is an important service that is supported by IoT. In today's time, waste management is a collective issue in most countries, which needs uninterrupted importance for management. In traditional waste management systems, the rapid growth of garbage leaves the public places unhygienic and dirty. The unhygienic environment can cause various deadly diseases. The prior research focused on the centralized system for waste management that is managed by a central authority. In this study, we are proposing a smart waste management system for real-time monitoring of "trash bins" in order to take timely actions for cleaning the bins and maintaining a disease-free environment for the people. The proposed system is based on edge-nodes, that is, trash bins. In this 


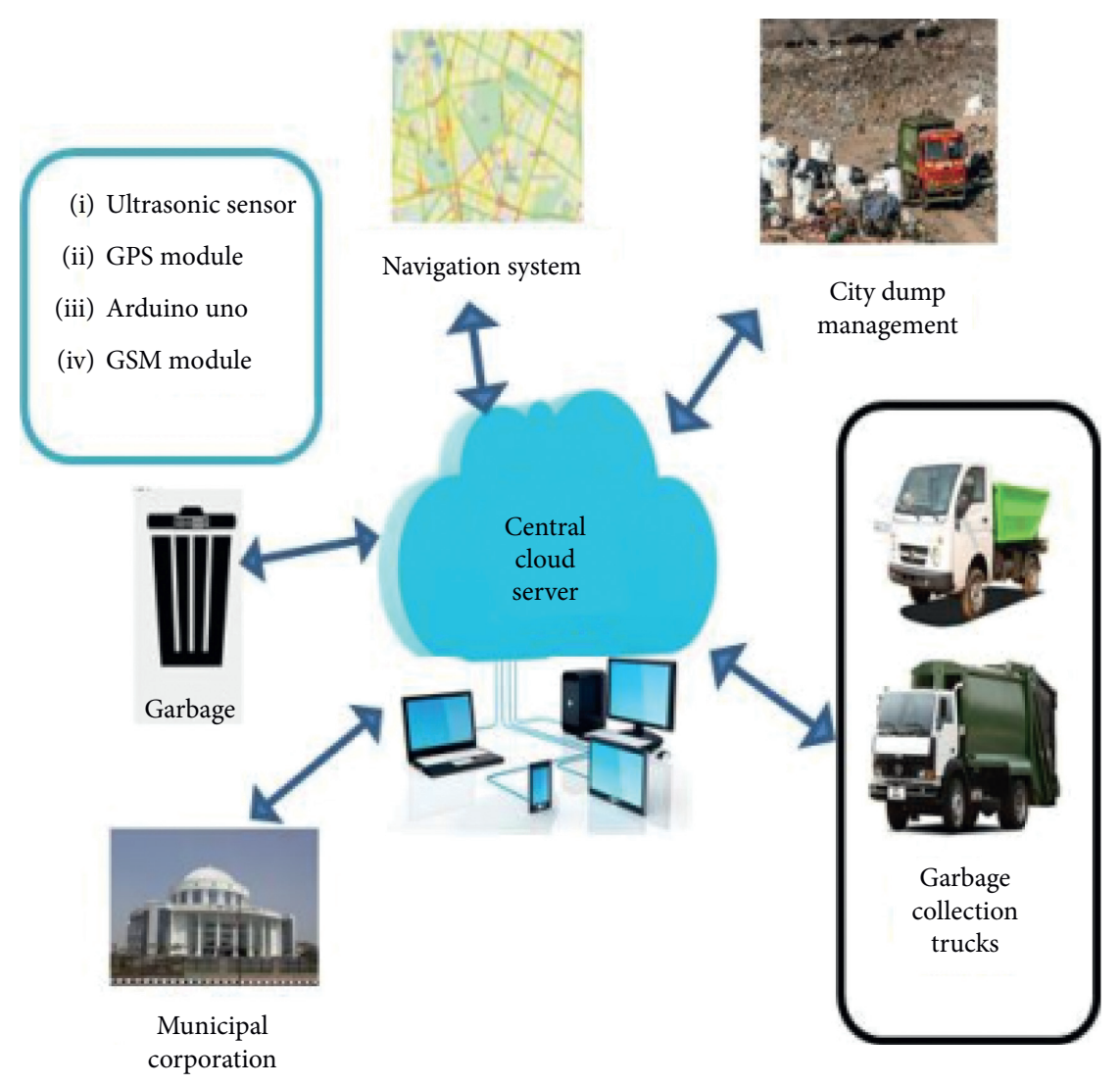

Figure 4: Architecture of SWC as a service.

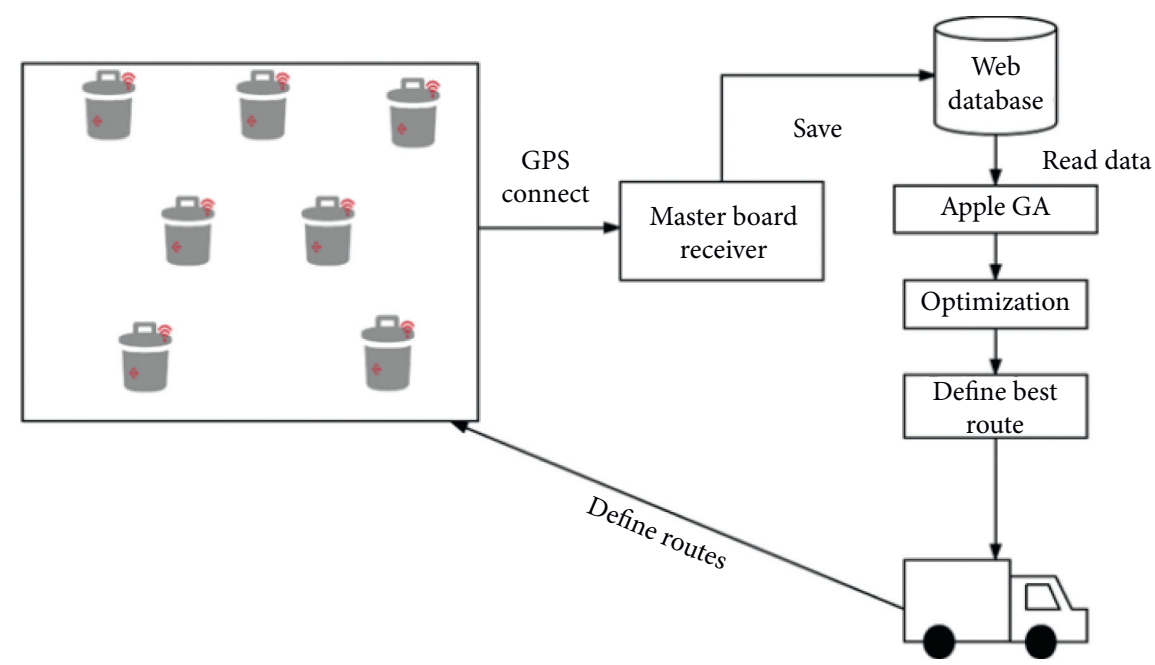

FigURE 5: System architecture of SCGCMS.

system, a trash bin is working as an intelligent node in the entire processing of waste management. The smart bin mechanism is illustrated in the form of a block diagram in Figure 6.

3.1. System Design. The proposed SBM system is composed of three main entities, that is, trash bins (TB), trash collecting vehicle (TCV), and central database (CDB). These entities are part of SBM and their duties and characteristics are defined in the following subsections.

3.1.1. Trash Bin. In SBM, a trash bin is an intelligent edgenode and a waste storage point in public areas. The trash bin provides the following information to the central database of the system: level of the bin (TBL) in percentage, color of the bin (TBC), and weight of the bin (TBW). Each trash bin has 
TABLe 1: Contributions and limitations of related schemes.

\begin{tabular}{|c|c|c|}
\hline Scheme & Contribution & Limitation \\
\hline SWMS [44] & Path optimization for garbage collecting van & Failure of sensors leads to system failure \\
\hline GMS [52] & $\begin{array}{l}\text { Fast transmitting mechanism for garbage } \\
\text { collection }\end{array}$ & Lacking in the reliability of communications \\
\hline IoT-based SWM [53] & Provision of statistical analysis & Lazy transportation affects all four entities \\
\hline $\begin{array}{l}\text { SWM by K-Query scheduling } \\
\text { [54] }\end{array}$ & $\begin{array}{c}\text { K-Query scheduling is used for database } \\
\text { management }\end{array}$ & $\begin{array}{l}\text { False monitoring can be a disadvantage for K-Query } \\
\text { scheduling }\end{array}$ \\
\hline SWC as a service [55] & Utilization of mobile app to facilitate van drivers & $\begin{array}{c}\text { Bandwidth constraints of cloud can affect the } \\
\text { mechanism }\end{array}$ \\
\hline SCGCMS [56] & Uses genetic algorithms for collecting waste & $\begin{array}{c}\text { Scalability issues can occur with complexity of the } \\
\text { system }\end{array}$ \\
\hline ML-based WMS [57] & $\begin{array}{l}\text { Flexibility and optimal path selection using } \\
\text { machine learning }\end{array}$ & Failure of batteries or interruption in solar provision \\
\hline
\end{tabular}

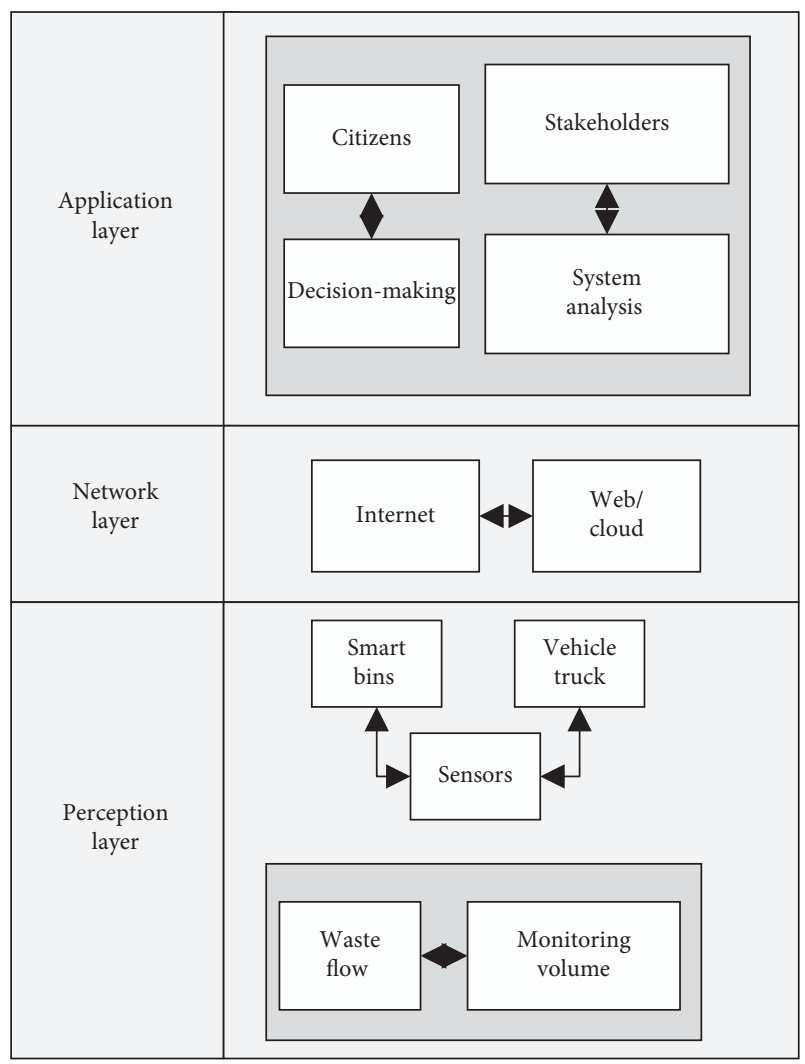

Figure 6: Block diagram of a smart bin mechanism.

its unique identification. Being a part of the IoT network, all trash bins are interconnected with each other through the Internet. Initially, each bin is green in color with level $<90 \%$. When the level of the bin increased to level $>=90 \%$, its color turns into red, which is the sign of a full bin.

3.1.2. Trash Collecting Vehicle. The vehicles that collect waste from the trash bins are dependent on the populated areas of smart cities. Mostly, smart cities are overpopulated which leads to difficulty in the collection of waste from densely populated areas using the same size of collecting vehicles. Each TCV is connected with the central database from where it gets information about the requesting TB. The
TCV collects waste from the trash bins and brings it to the dumping zones for further treatment.

3.1.3. Central Database. The central database is used as an information center as well as a storage point, which contains each and every detail of the TBs, TB-IDs, TCVs, their locations, and every single detail about these entities. Whenever an event occurs in the system, the processing information and status of trash bins are stored in the database.

3.2. Transmission Pattern of the Proposed System. The whole mechanism of collecting waste is described in Figure 7, adopted from [56]. When a trash bin gets filled or reaches its threshold limit, it changes its color into red and transmits a notification to the TCV through a gateway. The TB notification consists of TBL, TBC, and TBW. The TCV receives the request from the $\mathrm{TB}$ and forwards the status of the requesting $\mathrm{TB}$ to the database for updating. The TCV collects waste from the filled bin for further treatment, such as disposing waste or recycling and reusing waste. The TCV updates the status of requesting TB in the central database after collecting waste from the bin. The whole processing of the system is supported by Reduce, Reuse, and Recycle mechanisms.

3.3. Processing of the Trash Bin. The trash bin checks the level of waste. If the level $>=90 \%$, the TB changes its color into red and forwards a request to TCV for the cleaning process. If the level $<90 \%$, the TB color remains green, and without forwarding request, it rechecks its level and so on.

Generally, the proposed system is a repetitive mechanism that consists of the following steps: collecting waste, planning and analysis, segregating waste at the waste plant, and recycling or disposing of waste. The hardware structure of SBM consists of TBs, which are installed at different locations of the city having their unique IDs. At the initial level, each TB is green in color, while the weight and level of TBs are recorded accordingly. Once a TB reaches its threshold level, the color of that particular TB will change into red with obvious measurements of weight and level of waste in the percentage form. The TCV is another significant 


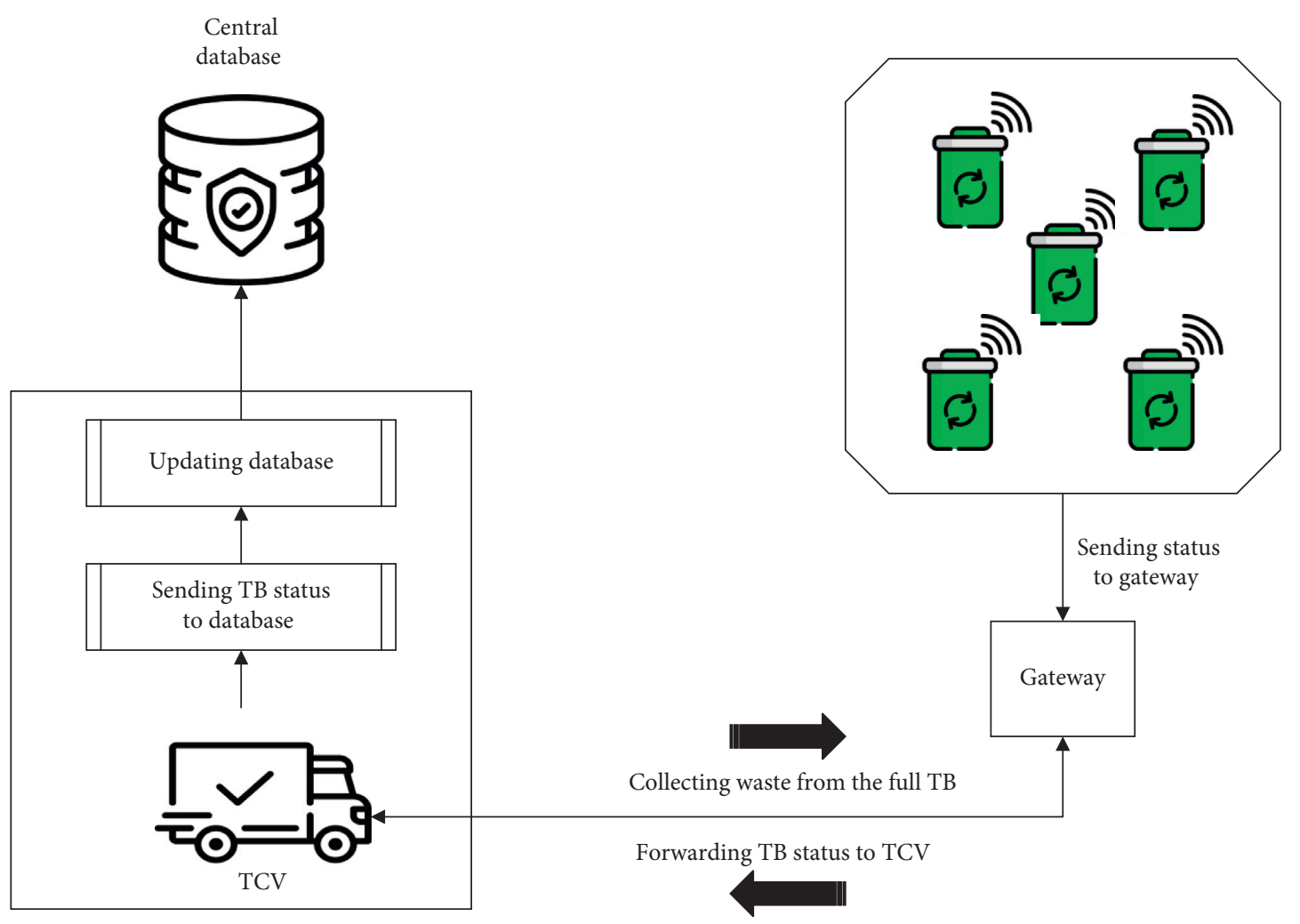

FIgURE 7: Transmission pattern of SBM.

factor in the proposed system. The TCV is informed by the filled TB to collect waste from it and updates the database with the new status of the requesting TB. The CDB is the central database in the SBM model. The duty of CDB is to store data regarding the location of the TB and TCV with the optimal route for waste collection. In SBM, for each event that occurred in the processing of waste collection and management, data for each process is also updated on the cloud. Eventually, the cloud contains information about each single event of the entities that are participating in the system.

Working of the SBM is described in the form of flow diagram, as shown in Figure 8. The main function of the proposed system is "trash to cash" that is based on three concepts, that is, Reduce, Recycle, and Reuse. "Reduce" refers to minimizing the amount of waste in the smart city as a particular situation of SBM. "Recycle" refers to recovering or reprocessing of dumped material that is extracted from the trash bins. "Reuse" refers to utilizing the dumped material after its recycling process. The processing of the system is started when an event is created in the trash bin. A threshold limit is set for each TB that helps easily accessing the bin and starts the cleaning process for quick and fast service provisioning.

3.4. Trash Bin Control Using Fuzzy Logic Processing. The fuzzy set theory plays a significant role in real-time scenarios to make decisions. Fuzzy logic was first introduced by Zadeh in 1965 [58]. The fuzzy logic is beneficial to deal with vagueness and uncertainty in real-time monitoring of the environment.

The fuzzy expert system (FES) is composed of three fundamental steps: fuzzification, inference rules, and defuzzification. A fuzzy expert system is the combined form of membership functions, if-then rules, and fuzzy operators. Moreover, FES is a mapping between the input and output values. Fuzzification converts crisp input values into fuzzy input, fuzzy rule base and/or knowledge base apply appropriate if-then rules, and defuzzification reconverts the fuzzy output into crisp output or in human readable form. The basic architecture of FES is presented in Figure 9.

In this study, fuzzy logic is utilized for decision-making in selecting appropriate locations to install trash bins. Fuzzy logic provides a better reading of the trash bin in real-time monitoring of the situation by using different levels of linguistic values. The linguistic variables have three levels of readings with different categories. We used three attributes for acquiring data of each trash bin, that is, TBL, TBC, and TBW, which indicate the trash bin level, color, and weight, respectively. Table 2 describes the input values of the trash bin level with categories low, medium, and high.

The input values for the attribute trash bin color are categorized into red, yellow, and green, and their ranges are defined in Table 3.

Table 4 shows the ranges of input values for trash bin weight with categories of light, medium, and heavy.

One attribute, that is, trash bin status (TBS), is used for the output readings of the trash bins. The TBS is classified 

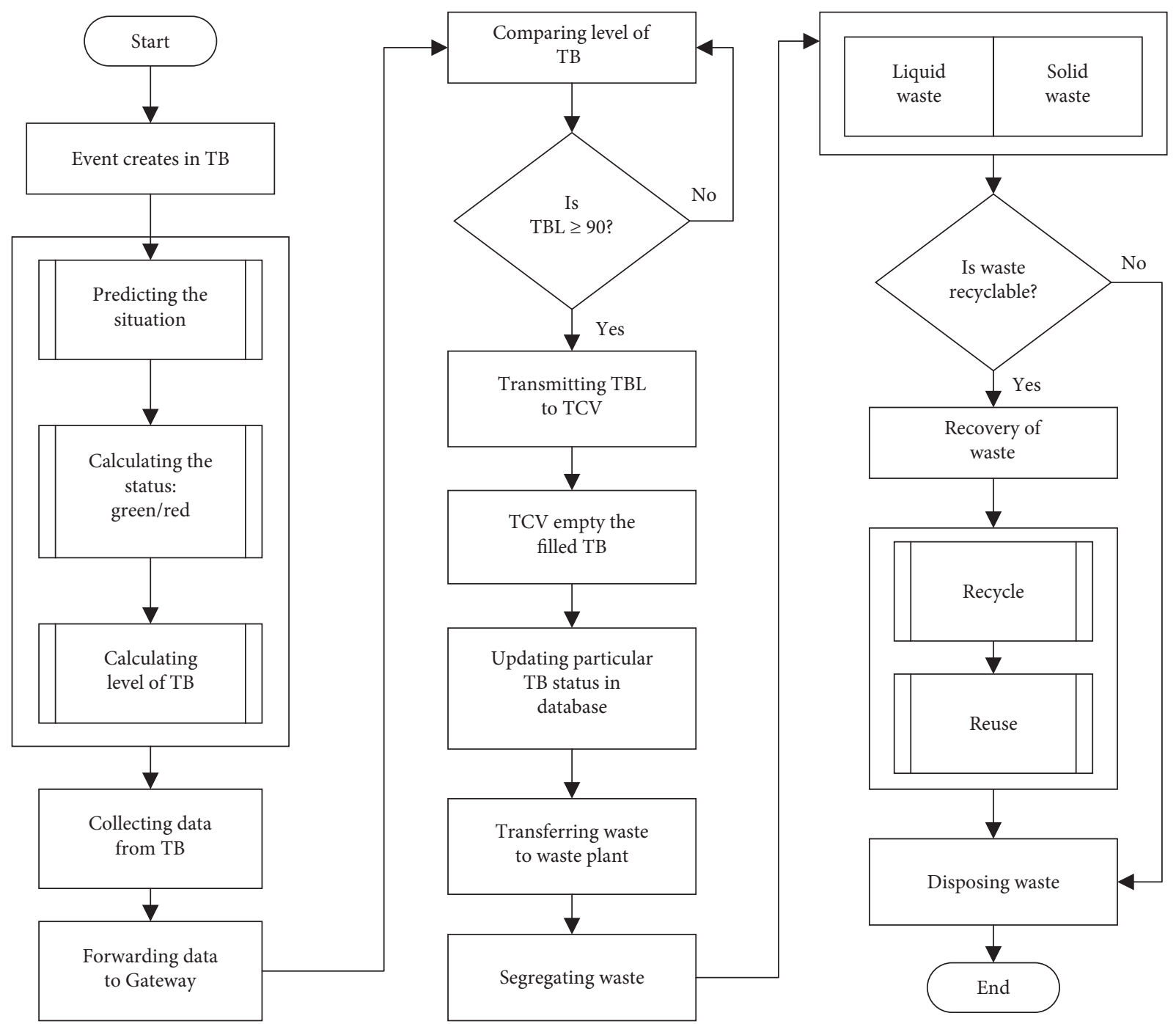

Figure 8: Functional flow diagram of the smart bin mechanism.

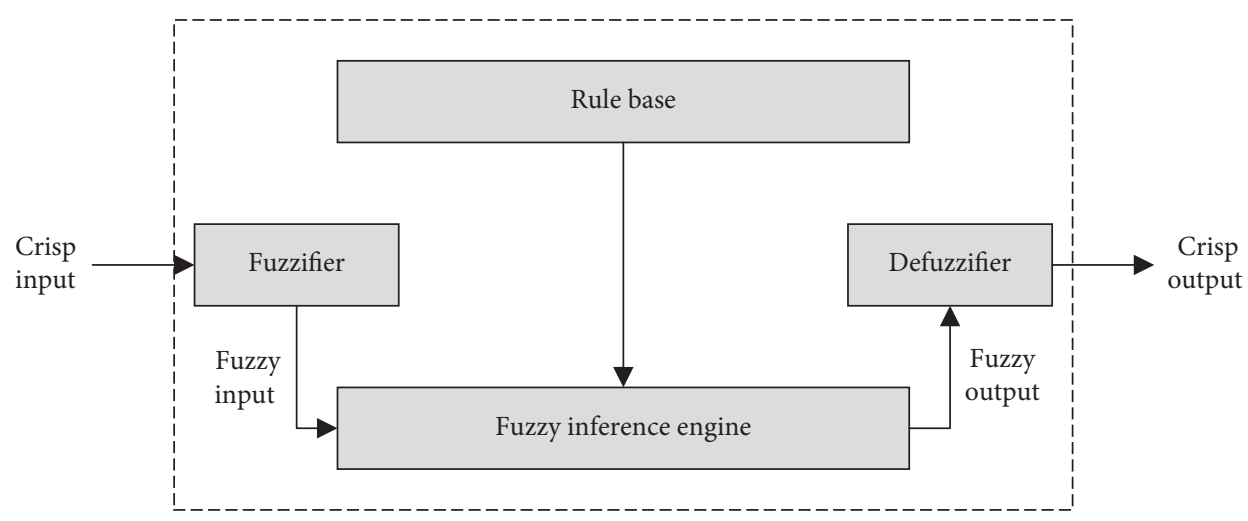

FIgURE 9: Fundamental architecture of FES.

into three stages, that is, bad, average, and good. The output ratings are described in Table 5 .

Fuzzy logic helps in decision-making in such types of scenarios while selecting appropriate locations and size for installing trash bins. In this scenario, two attributes, that is, access to heavy vehicles and distance from collecting points, are used for choosing suitable places to install trash bins. Based on these two attributes, the system makes decisions and these are divided into different categories described in Table 6. 
TABLE 2: Input ranges of the trash bin level (TBL).

\begin{tabular}{lcc}
\hline Categories & TBL ratings & Symbols \\
\hline Low & 0.1 to 0.5 & TBLL \\
Medium & 0.5 to 1.0 & TBLM \\
High & 1.0 to 1.5 & TBLH \\
\hline
\end{tabular}

TABLE 3: Input ranges of trash bin color (TBC).

\begin{tabular}{lcc}
\hline Categories & TBC ratings & Symbols \\
\hline Red & 0.1 to 0.2 & TBCR \\
Yellow & 0.2 to 0.4 & TBCY \\
Green & 0.4 to 1.0 & TBCG \\
\hline
\end{tabular}

TABLE 4: Input ranges of trash bin color (TBC).

\begin{tabular}{lcc}
\hline Categories & TBC ratings & Symbols \\
\hline Light & 0.1 to 0.6 & TBWL \\
Medium & 0.6 to 1.2 & TBWM \\
Heavy & 1.2 to 2.0 & TBWH \\
\hline
\end{tabular}

TABle 5: Output ranges of TB status (TBS).

\begin{tabular}{lcc}
\hline Categories & TBS ratings & Symbols \\
\hline Bad & $0 \%$ to $33 \%$ & TBSB \\
Average & $34 \%$ to $66 \%$ & TBSA \\
Good & $67 \%$ to $100 \%$ & TBSG \\
\hline
\end{tabular}

TABLE 6: Input values for selecting the site to install TB.

\begin{tabular}{lccc}
\hline Criteria & Location 1 & Location 2 & Location 3 \\
\hline Access for heavy vehicles & Medium & Difficult & Easy \\
$\begin{array}{l}\text { Distance from collecting } \\
\text { points }\end{array}$ & Small & Medium & Large \\
\hline
\end{tabular}

These parameters are helpful in selecting the best suitable site in the city to install trash bins for the citizens. The proposed system is reliable in terms of providing consistent services to the municipal department for monitoring and cleaning the city in real-time scenarios. For decision-making, the discussed attributes are used for generating fuzzy rules, such as if-then rules. These rules are then integrated to form an output value, which is demonstrated in Figure 10. Two input variables are used to choose a suitable location for the TB installation, which gives a single output by applying inference rules.

\section{Results and Discussion}

We have performed simulations of the proposed framework in real-life experimental environment with different test runs of loading and unloading of trash bins. The NetLogo platform is used to implement the smart waste management mechanism by using different simulation cases in time $T$ (minutes). At the initial stage, 20 to 25 trash bins are randomly distributed in a smart city with $\mathrm{TBL}=0 \%$ and $\mathrm{TBC}=$ green. On tick $1, \mathrm{TBL}=10 \%$ and $\mathrm{TBC}$ remains green . When $\mathrm{TBL}=90 \%$, TBC turns into red that is an alarming situation from the bin to a vehicle and thus the bin makes a request to the nearby collecting vehicle for the unloading process. For tick 1 , the simulation time $T=0$. In the first case, 10 trash bins are distributed in time $T=45$ minutes. Figure 11 shows the distribution of bins where the reading of each bin is recorded with different waste levels as per tick. The waste level in each trash bin is recorded against the total number of bins in the experimental environment. Each TB has some attributes, which are integrated to find the status/ readings of each bin. The process is shown in the following equation:

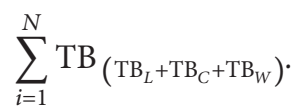

For a test run, one TB is selected as a testing bin and with waste level or level of filling, different rounds are performed on that particular bin. In Figure 12, 8 different rounds for a TB are shown that are directly corresponding to the level of filling the bin. These records show different readings on throwing action of garbage in a bin. The binunload operation is performed when a bin is full to its level. In Figure 12, $R$ denotes the readings of the selected testing bin.

The measuring weight level of a testing bin recorded with respect to time is described in Figure 13. It shows the weight of solid waste in a bin with regard to $\mathrm{kg}$ per time $\mathrm{T}$. It shows the readings of throwing waste as per operation or interval. The weight of waste/garbage that is thrown in the TB is measured in kilograms against the measured time $T$ in which a citizen throws it in the TB. 


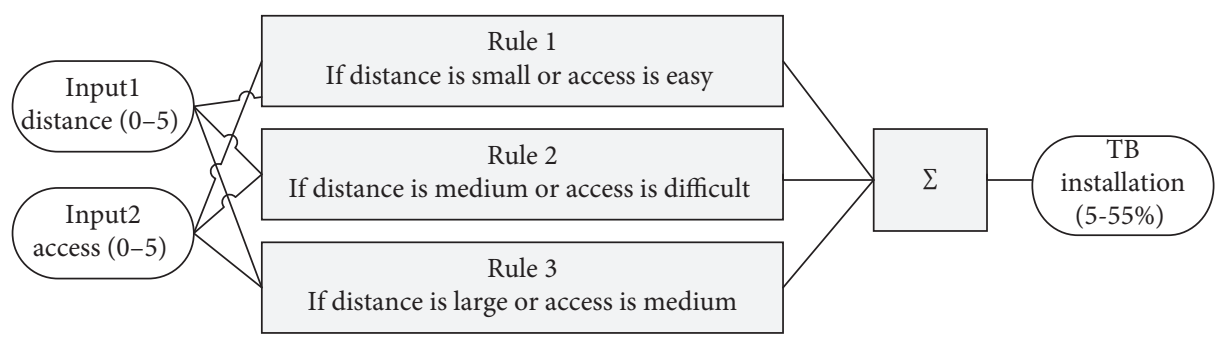

Figure 10: If-then rule to select appropriate locations for TB.

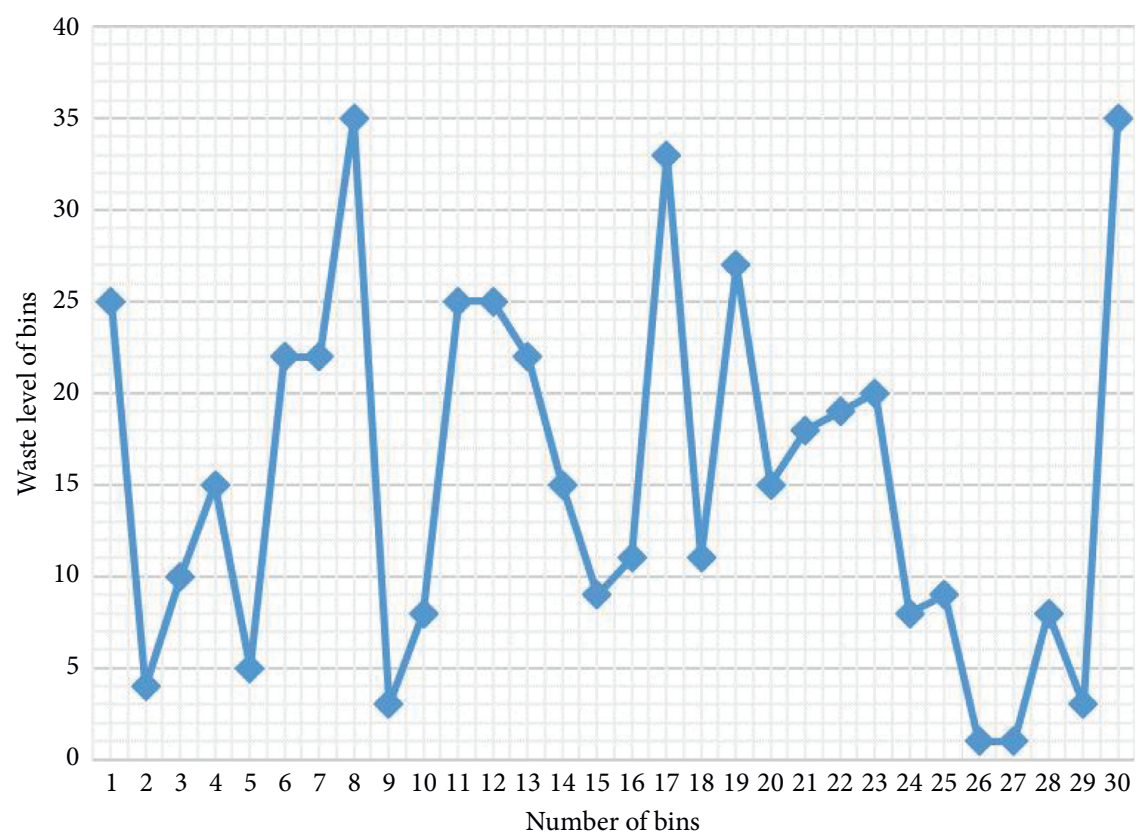

Figure 11: Readings of bins with different waste levels.

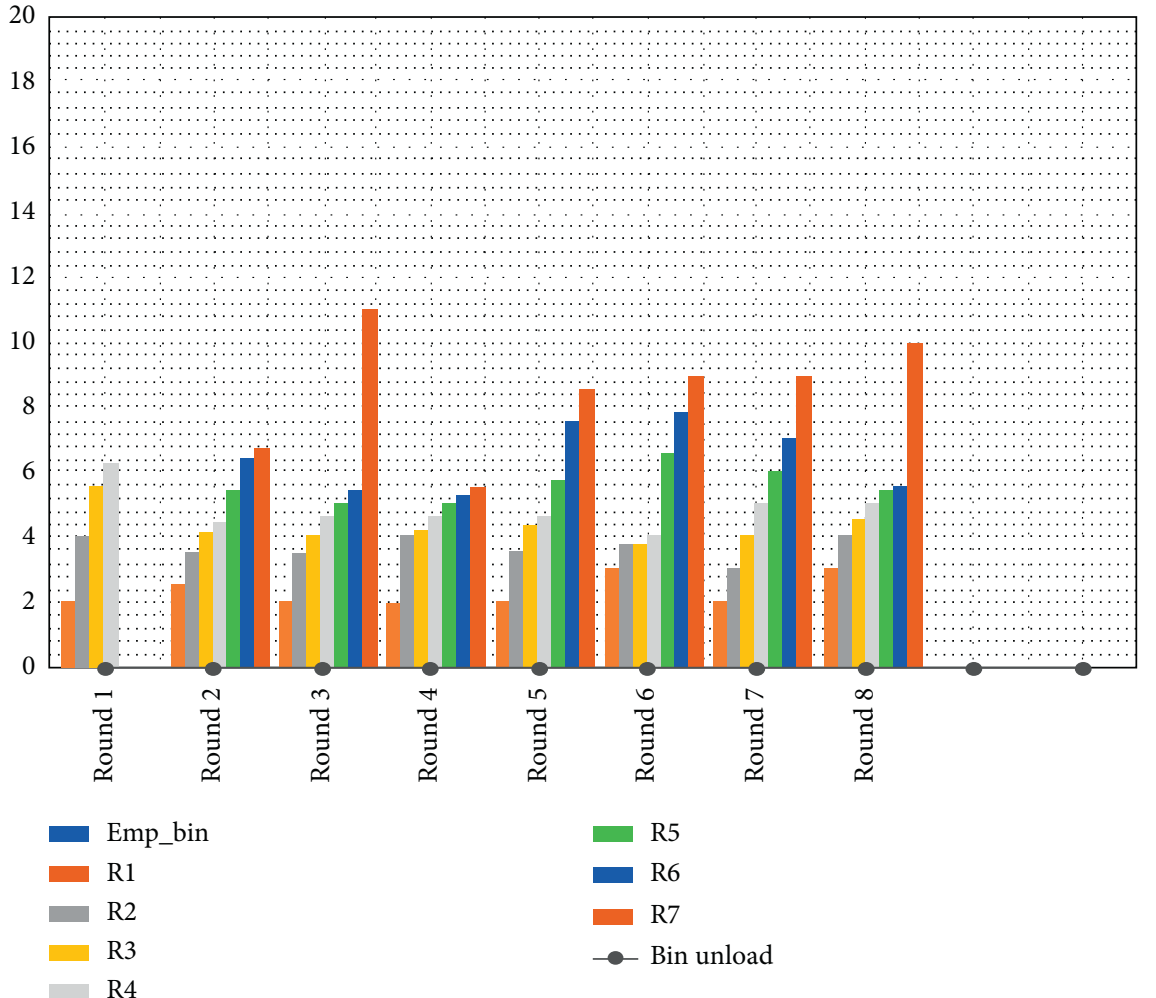

FIgURE 12: Readings of filling level with TB 1. 


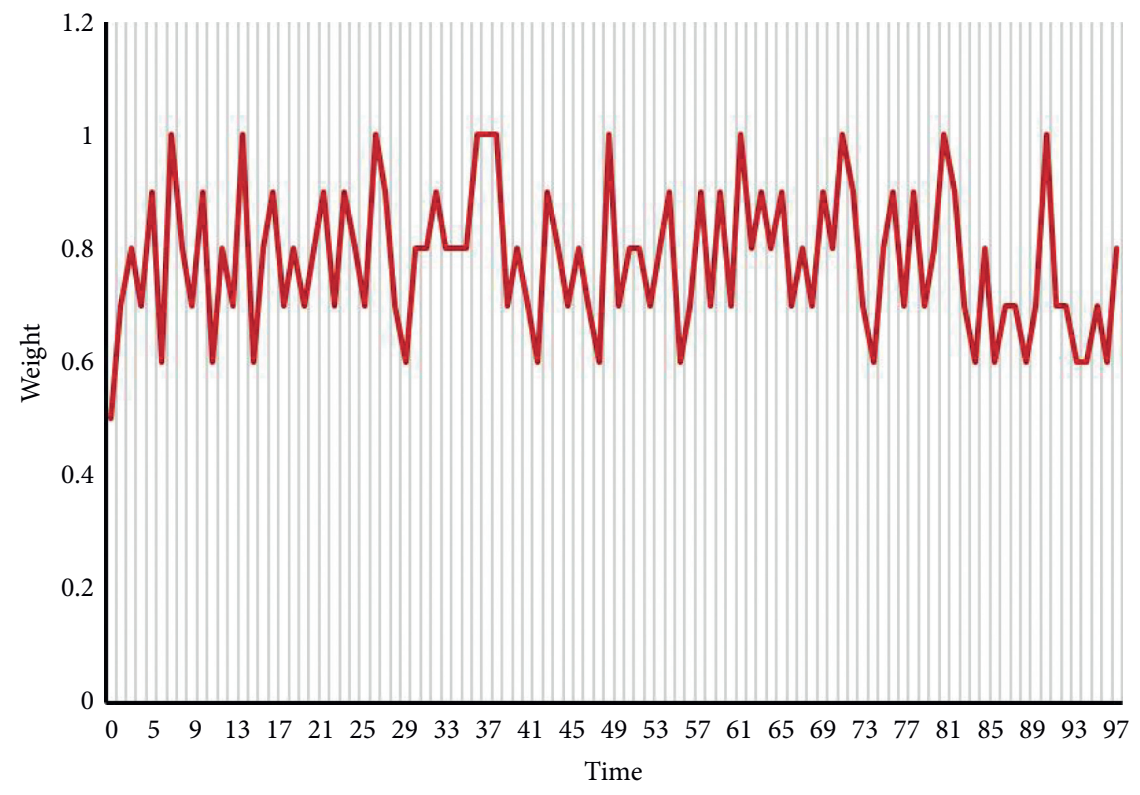

FIGURE 13: Measured weight of testing bin versus time $T$.

Two fuzzy parameters are used to decide an appropriate location for installing trash bins in the city. Fuzzy parameters are helpful in selecting the size of the trash bins depending on the density of the area, such as less, moderate, and high dense areas.

\section{Conclusion}

Conventional solid waste management systems have several shortcomings in terms of late unloading, hindrance in new techniques, lacking in throughput, less access to actual data, and many more. Therefore, an advanced approach is the need for the time to overcome all existing problems in the waste collecting process. Generally, waste collection has more consumption of cost from the municipality budget. In this paper, a real-time smart bin monitoring framework is proposed to get real-time access to data from the bins and implement the collecting procedure accordingly. The proposed framework is achieved by using a theoretical and architectural model. The model is implemented in a real-time environment of NetLogo and the experimental results show that the proposed framework is very responsive and effective for the environment. It is also effective for the economical aspects as it reduces the cost of labors and fuel cost of collecting vehicles by minimizing their extra visits in checking bins' status. Once a bin reaches its threshold limit, it informs the collecting vehicle for the cleaning process, which saves time, cost, and energy. The SBM is userfriendly as it obstructs the overflow of bins. It is useful for IoTbased smart cities, which helps to keep the environment clean and disease-free for the citizens. The SBM is supportive for realtime scenarios by using fuzzy logic processing in order to designate trash bins according to space and density of the environment in public areas. Fuzzy logic helps the system in selecting the best fitted site for each trash bin. Generally, fuzzy logic boosts the system for performing effectively in the environment.

\section{Challenges and Future Work}

Though the SBM framework designed for smart cities in the context of IoT has potentials, at the same time, it has the following challenges:

(i) Distribution of trash bins in the most populated areas where the amount of waste is unpredictable on daily basis

(ii) Disturbance in the Internet connectivity due to various causes, that is, weather disruption or defected connection

(iii) Lazy transportation: traffic jam could be a big challenge for vehicles to reach on time and collect garbage

(iv) Communication between two entities and damage of batteries could be severe challenges for the system

In the future, the model may be extended to an alternate and the shortest pathfinding for collecting vehicles in order to enhance transportation and remove collecting barriers. In addition, adding alternate sources for connectivity in case of power failure or weather hindrance may also be considered. Further, to facilitate the mechanism and save more energy, automated segregating TBs can be installed for dry, wet, and hazardous types of waste.

\section{Data Availability}

This research is based on simulations, which are performed in a simulator. Therefore, there is no dataset used in this research.

\section{Conflicts of Interest}

The authors declare that they have no conflicts of interest. 


\section{Acknowledgments}

This work was supported by King Saud University, Saudi Arabia, through Research Supporting Project no. RSP-2020/ 186.

\section{References}

[1] C. Srinivasan, B. Rajesh, P. Saikalyan, K. Premsagar, and E. S. Yadav, "A review on the different types of internet of things (IOT)," Journal of Advanced Research in Dynamical and Control Systems, vol. 11, no. 1, pp. 154-158, 2019.

[2] K. Ashton, "That "internet of things" thing," RFID Journal, vol. 22, no. 7, pp. 97-114, 2009.

[3] C. Perera, A. Zaslavsky, P. Christen, and D. Georgakopoulos, "Context aware computing for the internet of things: a survey," IEEE Communications Surveys \& Tutorials, vol. 16, no. 1, pp. 414-454, 2014.

[4] L. Wang, Y. Ali, S. Nazir, and M. Niazi, "Isa evaluation framework for security of internet of health things system using ahp-topsis methods," IEEE Access, vol. 8, pp. 152316-152332, 2020.

[5] A. Tahir, F. Chen, H. U. Khan et al., "A systematic review on cloud storage mechanisms concerning e-healthcare systems," Sensors, vol. 20, no. 18, p. 5392, 2020.

[6] A. Ahmad, A. Ullah, C. Feng et al., "Towards an improved energy efficient and end-to-end secure protocol for iot healthcare applications," Security and Communication Networks, vol. 2020, Article ID 8867792, 10 pages, 2020.

[7] A. J. Jara, M. A. Zamora, and A. F. G. Skarmeta, "An internet of things-based personal device for diabetes therapy management in ambient assisted living (AAL)," Personal and Ubiquitous Computing, vol. 15, no. 4, pp. 431-440, 2011.

[8] M. Sikarndar, W. Anwar, A. Almogren, I. Ud Din, and N. Guizani, "Iomt-based association rule mining for the prediction of human protein complexes," IEEE Access, vol. 8, pp. 6226-6237, 2020.

[9] K. A. Awan, I. U. Din, A. Almogren, H. Almajed, I. Mohiuddin, and M. Guizani, "NeuroTrust-artificial neural network-based intelligent trust management mechanism for large-scale internet of medical things," IEEE Internet of Things Journal, In press.

[10] A. Almogren, I. Mohiuddin, I. U. Din, H. Al Majed, and N. Guizani, "FTM-IoMT: fuzzy-based trust management for preventing sybil attacks in internet of medical things," IEEE Internet of Things Journal, In press.

[11] R. Wirza, S. Nazir, H. U. Khan, I. García-Magariño, and R. Amin, "Augmented reality interface for complex anatomy learning in the central nervous system: a systematic review," Journal of Healthcare Engineering, vol. 2020, Article ID 8835544, 15 pages, 2020.

[12] S. R. Khan, M. Sikandar, A. Almogren, I. Ud Din, A. Guerrieri, and G. Fortino, "IoMT-based computational approach for detecting brain tumor," Future Generation Computer Systems, vol. 109, pp. 360-367, 2020.

[13] H. Chen, S. Khan, B. Kou, S. Nazir, W. Liu, and A. Hussain, "A smart machine learning model for the detection of brain hemorrhage diagnosis based internet of things in smart cities," Complexity, vol. 2020, Article ID 3047869, 10 pages, 2020.

[14] A. Zanella, N. Bui, A. Castellani, L. Vangelista, and M. Zorzi, "Internet of things for smart cities," IEEE Internet of Things Journal, vol. 1, no. 1, pp. 22-32, 2014.

[15] H. A. Khattak, K. Tehreem, A. Almogren, Z. Ameer, I. U. Din, and M. Adnan, "Dynamic pricing in industrial internet of things: blockchain application for energy management in smart cities," Journal of Information Security and Applications, vol. 55, p. 102615, 2020.

[16] S. D. T. Kelly, N. K. Suryadevara, and S. C. Mukhopadhyay, "Towards the implementation of iot for environmental condition monitoring in homes," IEEE Sensors Journal, vol. 13, no. 10, pp. 3846-3853, 2013.

[17] M. T. Lazarescu, "Design of a WSN platform for long-term environmental monitoring for iot applications," IEEE Journal on Emerging and Selected Topics in Circuits and Systems, vol. 3, no. 1, pp. 45-54, 2013.

[18] S. Tozlu, M. Senel, W. Wei Mao, and A. Keshavarzian, "Wi-fi enabled sensors for internet of things: a practical approach," IEEE Communications Magazine, vol. 50, no. 6, pp. 134-143, 2012.

[19] W. Ali, I. U. Din, A. Almogren, M. Guizani, and M. Zuair, “A lightweight privacy-aware iot-based metering scheme for smart industrial ecosystems," IEEE Transactions on Industrial Informatics, In presss.

[20] L. Foschini, T. Taleb, A. Corradi, and D. Bottazzi, "M2mbased metropolitan platform for IMS-enabled road traffic management in iot," IEEE Communications Magazine, vol. 49, no. 11 , pp. $50-57,2011$.

[21] K. A. Awan, I. U. Din, A. Almogren, M. Guizani, and S. Khan, "StabTrust-a stable and centralized trust-based clustering mechanism for iot enabled vehicular ad-hoc networks," IEEE Access, vol. 8, pp. 21159-21177, 2020.

[22] F. M. Malik, H. A. Khattak, A. Almogren, O. Bouachir, I. U. Din, and A. Altameem, "Performance evaluation of data dissemination protocols for connected autonomous vehicles," IEEE Access, vol. 8, pp. 126896-126906, 2020.

[23] S. K. Tayyaba, H. A. Khattak, A. Almogren et al., "5G vehicular network resource management for improving radio access through machine learning," IEEE Access, vol. 8, pp. 6792$6800,2020$.

[24] G. Jadoon, I. Ud Din, A. Almogren, and H. Almajed, "Smart and agile manufacturing framework, a case study for automotive industry," Energies, vol. 13, no. 21, p. 5766, 2020.

[25] Z. Ali, M. A. Shah, A. Almogren, I. Ud Din, C. Maple, and H. A. Khattak, "Named data networking for efficient iot-based disaster management in a smart campus," Sustainability, vol. 12, no. 8, p. 3088, 2020.

[26] K. Haseeb, I. Ud Din, A. Almogren, and N. Islam, “An energy efficient and secure IoT-based WSN framework: an application to smart agriculture," Sensors, vol. 20, no. 7, p. 2081, 2020.

[27] K. A. Awan, I. Ud Din, A. Almogren, and H. Almajed, "AgriTrust-a trust management approach for smart agriculture in cloud-based internet of agriculture things," Sensors, vol. 20, no. 21, p. 6174, 2020.

[28] W. Ali, I. U. Din, A. Almogren, and N. Kumar, "Alpha: an anonymous orthogonal code-based privacy preserving scheme for industrial cyber physical systems," IEEE Transactions on Industrial Informatics, In press.

[29] I. U. Din, M. Guizani, S. Hassan et al., "The internet of things: a review of enabled technologies and future challenges," IEEE Access, vol. 7, pp. 7606-7640, 2018.

[30] I. U. Din, M. Guizani, J. J. P. C. Rodrigues, S. Hassan, and V. V. Korotaev, "Machine learning in the internet of things: designed techniques for smart cities," Future Generation Computer Systems, vol. 100, pp. 826-843, 2019.

[31] K. Haseeb, A. Almogren, I. Ud Din, N. Islam, and A. Altameem, "SASC: secure and authentication-based sensor 
cloud architecture for intelligent internet of things," Sensors, vol. 20 , no. 9, p. 2468, 2020.

[32] K. Haseeb, I. Ud Din, A. Almogren, N. Islam, and A. Altameem, "RTS: a robust and trusted scheme for iot-based mobile wireless mesh networks," IEEE Access, vol. 8, pp. 68379-68390, 2020.

[33] B. Liao, Y. Ali, S. Nazir, L. He, and H. U. Khan, "Security analysis of iot devices by using mobile computing: a systematic literature review," IEEE Access, vol. 8, pp. 120331-120350, 2020.

[34] K. Haseeb, N. Islam, A. Almogren, and I. U. Din, "Intrusion prevention framework for secure routing in WSN-based mobile internet of things," Ieee Access, vol. 7, pp. 185496-185505, 2019.

[35] K. A. Awan, I. U. Din, A. Almogren, M. Guizani, A. Altameem, and S. U. Jadoon, "RobustTrust-a pro-privacy robust distributed trust management mechanism for internet of things," IEEE Access, vol. 7, pp. 62095-62106, 2019.

[36] K. S. Awaisi, A. Abbas, M. Zareei et al., "Towards a fog enabled efficient car parking architecture," IEEE Access, vol. 7, pp. 159100-159111, 2019.

[37] I. U. Din, A. Almogren, M. Guizani, and M. Zuair, "A decade of internet of things: analysis in the light of healthcare applications," IEEE Access, vol. 7, pp. 89967-89979, 2019.

[38] N. Islam, K. Haseeb, A. Almogren, I. U. Din, M. Guizani, and A. Altameem, "A framework for topological based map building: a solution to autonomous robot navigation in smart cities," Future Generation Computer Systems, vol. 111, pp. 644-653, 2020.

[39] B. Jan, H. Farman, M. Khan, M. Talha, and I. U. Din, "Designing a smart transportation system: an internet of things and big data approach," IEEE Wireless Communications, vol. 26, no. 4, pp. 73-79, 2019.

[40] C. Zhu, V. C. Leung, L. Shu, and E. C.-H. Ngai, "Green internet of things for smart world," IEEE Access, vol. 3, pp. 2151-2162, 2015.

[41] S. U. Islam, H. A. Khattak, J.-M. Pierson et al., "Leveraging utilization as performance metric for CDN enabled energy efficient internet of things," Measurement, vol. 147, p. 106814, 2019.

[42] K. Janjua, M. A. Shah, A. Almogren, H. A. Khattak, C. Maple, and I. U. Din, "Proactive forensics in IoT: privacy-aware logpreservation architecture in fog-enabled-cloud using holochain and containerization technologies," Electronics, vol. 9, no. 7, p. 1172, 2020.

[43] R. Liu and J. Wang, "Internet of things: application and prospect," in Proceedings of the MATEC Web of Conferences, p. 02034, 2017.

[44] S. Mahajan, A. Kokane, A. Shewale, M. Shinde, and S. Ingale, "Smart waste management system using iot," International Journal of Advanced Engineering Research and Science, vol. 4, no. 4, 2017.

[45] H. A. Khattak, H. Farman, B. Jan, and I. U. Din, "Toward integrating vehicular clouds with iot for smart city services," IEEE Network, vol. 33, no. 2, pp. 65-71, 2019.

[46] C. Balakrishna, "Enabling technologies for smart city services and applications," in Proceedings of the 2012 Sixth International Conference on Next Generation Mobile Applications, Services and Technologies, pp. 223-227, IEEE, Paris, France, September 2012.

[47] H. Khattak, Z. Ameer, U. Din, and M. Khan, "Cross-layer design and optimization techniques in wireless multimedia sensor networks for smart cities," Computer Science and Information Systems, vol. 16, no. 1, pp. 1-17, 2019.
[48] S. U. Khan, N. Islam, Z. Jan, I. U. Din, A. Khan, and Y. Faheem, "An E-health care services framework for the detection and classification of breast cancer in breast cytology images as an IoMT application," Future Generation Computer Systems, vol. 98, pp. 286-296, 2019.

[49] A. Siddiqua, M. A. Shah, H. A. Khattak, I. Ud Din, and M. Guizani, "ICAFE: intelligent congestion avoidance and fast emergency services," Future Generation Computer Systems, vol. 99, pp. 365-375, 2019.

[50] T. Anagnostopoulos, A. Zaslavsky, K. Kolomvatsos et al., "Challenges and opportunities of waste management in iotenabled smart cities: a survey," IEEE Transactions on Sustainable Computing, vol. 2, no. 3, pp. 275-289, 2017.

[51] O. A. Khan, M. A. Shah, I. U. Din et al., "Leveraging named data networking for fragmented networks in smart metropolitan cities," IEEE Access, vol. 6, pp. 75899-75911, 2018.

[52] M. H. A. Gawad, M. D. Katkoria, M. S. Kadam, and M. D. T. Jain, "Garbage monitoring system using IOT," International Journal of Engineering Sciences \& Research Technology, vol. 7, pp. 319-324, 2018.

[53] N. Abdullah, O. A. Alwesabi, and R. Abdullah, "Iot-based smart waste management system in a smart city," in Proceedings of the International Conference of Reliable Information and Communication Technology, pp. 364-371, Springer, Kuala Lumpur, Malaysia, July 2018.

[54] F. M. Hadria, S. Jayanthy, A. Arunraja, and E. E. Vigneswaran, "Iot based smart waste management using top k-query scheduling," in Proceedings of the 2018 Second International Conference on Intelligent Computing and Control Systems (ICICCS), pp. 448-452, IEEE, Madurai, India, July 2018.

[55] S. S. Chaudhari and V. Y. Bhole, "Solid waste collection as a service using iot-solution for smart cities," in Proceedings of the 2018 International Conference on Smart City and Emerging Technology (ICSCET), pp. 1-5, IEEE, Mumbai, India, January 2018.

[56] M. S. Chaudhari, B. Patil, and V. Raut, "Iot based waste collection management system for smart cities: an overview," in Proceedings of the 2019 3rd International Conference on Computing Methodologies and Communication (ICCMC), pp. 802-805, IEEE, Erode, India, March 2019.

[57] T. Anh Khoa, C. H. Phuc, P. D. Lam et al., "Waste management system using iot-based machine learning in university," Wireless Communications and Mobile Computing, vol. 2020, Article ID 6138637, 13 pages, 2020.

[58] L. A. Zadeh, "A computational approach to fuzzy quantifiers in natural languages," Computers \& Mathematics with Applications, vol. 9, no. 1, pp. 149-184, 1983. 